Narges Sadeghi', Fatemeh Etedali², Mahboubeh Firouzkouhi Moghadam³, Alireza Shamsi4

${ }^{1}$ Community Health Research Center, Isfahan (Khorasgan) Branch, Islamic Azad University, Isfahan, Iran

IIsfahan (Khorasgan) Branch, Islamic Azad University, Isfahan, Iran

${ }^{3}$ Department of Psychiatry, Research Center for Children and Adolescents Health (RCCAH),

Zahedan University of Medical Sciences, Zahedan, Iran

${ }^{4}$ School of Medicine, Shahid Beheshti University of Medical Sciences, Tehran, Iran

\title{
Comparison of behavioral problems between the children with one schizophrenic parent and children with healthy parents
}

\begin{abstract}
Introduction: The quality of the relationship between children and parents in the early years of life is one of the most effective factors in the mental growth of the children. A parent being affected by schizophrenia may have very deep effects on the mental growth of the children. This study aimed to compare the behavioral problems in children with one schizophrenic parent and children with healthy parents.

Material and methods: This descriptive study was performed in Zahedan, Iran in 2014-2015. The behavioral problems of 60 children with a schizophrenic parent were compared to that of 60 children with healthy parents. The Child Behavior Checklist (CBCL) was used to evaluate the two groups.

Results: The comparison of the $C B C L$ score between two groups showed a significant difference $(P<0.005)$. Moreover, the $C B C L$ score of primary school girls $(P=0.03)$ and boys $(P=0.04)$ was significantly different between the two groups. No significant difference was reported between the two groups regarding the $C B C L$ score of teenage girls $(P=0.09)$ and teenage boys $(P=0.09)$.

Conclusions: Our findings can be regarded as evidence supporting the effect of psychotic disorders, such as schizophrenia in parents on the behavioral problems of children.
\end{abstract}

Psychiatry 2020; 17, 3: 109-114

Key words: children, behavioral problems, parents, schizophrenia

\section{Introduction}

The quality of the relationship with parents is one of the main predictors of cognitive, emotional and social development of children. It is recognized as one of the leading factors for improving the subjective well-being features of children, including happiness and life satisfaction [1] Moreover, adolescence is a vulnerable period for developing depression, particularly among girls [2]. The quality of the parent-adolescent relationship might be considered

Adres do korespondencji:

Alireza Shamsi

School of Medicine

Shahid Beheshti University

of Medical Sciences, Tehran, Iran

e-mail: ar.shamsi@sbmu.ac.ir as an index for adolescent depressive symptoms [3]. Parent-adolescent relationship quality was assessed in a study performed by Branje et al. [2], which introduced this factor as a predictor for depression symptoms in adolescents. In other words, depression symptoms were predictable by evaluating the quality of relationships with mothers in both genders. In addition, the quality of relationships with fathers was of importance in boys. Furthermore, childhood emotional deprivation, parental unresponsiveness to the child, and distance between parents and children are among the factors influencing the psychological status of children. In addition, the lack of support to meet the needs of children, proper interaction between parents and children, and common 
sympathy in stress conditions are known as the main factors leading to the occurrence of behavioral problems and disorders [4].

Behavioral issues affect all the aspects of an individual, as well as the social life of children. These complications usually occur for the first time in middle childhood and adolescence with a peak in the age of 8-15 years [5]. Consequently, various studies focused on the behavioral problems of middle childhood and adolescence.

The literature shows that some characteristics, for example internalizing or externalizing behaviors, emotional adjustment, self-regulation, and substance use may be affected by defective interactions between children and parents [6]. These problems are strongly affected by family factors, such as parental depression [7], marital distress [8], parenting practices [9], and parent-child relationships [9]. Neece et al. [6] evaluated the relationship between parenting stress and child behavior problems evaluated in 237 children. These authors demonstrated a bidirectional relationship between these factors.

In recent years, behavioral problems in children and adolescents have grown dramatically resulting in diverse complications in the field of individual interactions. Moreover, behavioral problems exert a remarkable effect on the educational status and social promotion of children. Considering the negative impacts of behavioral problems on the development of children, the investigation of factors forming this phenomenon is of high importance. The family has been confirmed to have a great impact on the behavioral problems of children. The role of the family in the growth and progress of children is conceptually explained by the attachment theory of John Bally [10] and Mary Ainsworth [11]. According to their theory, the child's experience in the family accounts for the nature of the relationships of children with important people of their lives, as well as many aspects of the social, emotional, and cognitive development in the early years [11].

Schizophrenia is a severe mental disorder, which presents with psychotic symptoms usually in adolescence or in early adulthood [12]. Based on DSM-IV-TR criteria, the annual incidence of schizophrenia varies from 0.5 to 5 cases in every 10,000 people with roughly the same incidence and prevalence throughout the world. The prevalence of schizophrenia is equal between both genders; however, the morbidity of the disease is different [12].

In recent years, researchers have taken into consideration the at-risk populations, namely the children and adolescents of parents with schizophrenia. Emotional and cognitive problems have been found to be the main issues in the first-degree relatives of people with schizophrenia [13].
The presence of this disorder in parents can impose an impact on the personality and performance of children in the future. The current study aimed to compare the behavioral problems between the offspring with one parent affected by schizophrenia and children with healthy parents.

\section{Material and methods}

This case-control descriptive study was performed in Zahedan, Iran in 2014-2015. The behavioral problems of 60 children with a parent suffering from schizophrenia referring to the Baharan Psychiatric Hospital (Zahedan, Iran) were compared to that of 60 children with healthy parents referring to Ali ibn Abi Talib General Hospital (Zahedan, Iran).

\section{Inclusion and Exclusion Criteria}

The inclusion criteria entailed being 6-18 years old, having one schizophrenic parent diagnosed for at least two years based on DSM-IV-TR, another parent being healthy, living with two parents, and willingness for participation in the study. The exclusion criteria encompassed being affected by psychiatric disorders other than schizophrenia, chronic physical illness, and mental retardation.

\section{Study Design}

This study was conducted after obtaining the required permissions from the Faculty of Nursing and Midwifery, Islamic Azad University of Isfahan (Khorasgan). The present investigation was performed at the clinics of Baharan Psychiatric Hospital and Ali ibn Abi Talib General Hospital, Zahedan, Iran.

A total of 120 children and adolescents were selected through target sampling and were divided into two matched groups of 60 cases each. The two groups were matched in terms of demographic characteristics. The behavioral problems of children with a schizophrenic parent referring to the Psychiatry Hospital were compared with children who had healthy parents referring to General Hospital.

\section{Child Behavior Checklist (CBCL)}

The CBCL was used as the data collection tool. This 113-item questionnaire is utilized to assess the emotional, behavioral, social, mental, and attention problems of children in two age groups of children (4-11 years) and adolescents (12-18 years old). This scale is used as an important screening tool for psychiatric disorders in the age group of 4-18 years.

This checklist is completed by parents or people who are responsible to take care of the child. The scores of this test are categorized in the three domains of internal- 
izing problems (including somatic complaints, anxiety, depression, and withdrawn), externalizing problems (including rule-breaking and aggressive behaviors), and general problems. The score is derived applying all the mentioned subscales along with 33 additional items examining other problems.

The score of this checklist ranges from 1-100 with a lower score indicating a better situation. In addition, the standardized score has a mean of $50 \pm 10$ with higher scores representing more behavioral problems. The reliability coefficient of this checklist is 0.75 [14]. The sensitivity and specificity of the test have been calculated as 0.79 and 0.98 in Iran, respectively [15].

\section{Statistical analysis}

All the data were analyzed by SPSS software version 18 . Descriptive statistics were utilized to report the frequency of all the variables. In order to compare the two groups, the t-test and Chi-square test were used. A P-value of less than 0.05 was considered significant for all tests.

\section{Ethical considerations}

The present investigation was approved by the Islamic Azad University of Isfahan, Khorasgan Branch. All the participants were assured in terms of data confidentiality and informed consent was obtained from all subjects. To follow the ethical considerations, all the stages of the study and research techniques were explained to the individuals. The participants were assured that they could leave the research in case of an unwillingness for continuing the study.

\section{Results}

Our findings showed that the mean age of children in the group of children with a schizophrenic parent and the control group was $10.87 \pm 3.32$ (range of 4.5-18) and $10.12 \pm 3.23$ (range of 4-18) years, respectively. The comparisons showed that there was no significant difference between the two groups regarding the mean age of the children $(P>0.05)$. The frequency of age and gender in the two groups is shown in Table 1.
The mean of the $C B C L$ score in the group of schizophrenic parents and the control group was $61.5 \pm 12.06$ (range of 39-87) and $51.61 \pm 11.54$ (range of 30-69), respectively. According to the results of the Kolmogorov-Smirnov test, the distribution of the $C B C L$ score was normal $(P=$ 0.28). The homoscedasticity of the $C B C L$ score was analyzed by Levene's test, which confirmed the homogeneity of the $C B C L$ score variances $(F=0.01 ; P=0.63)$. The comparison of the $\mathrm{CBCL}$ score between the case and control groups revealed a significant difference $(P<0.005)$. The mean and results of comparing the $\mathrm{CBCL}$ score in terms of gender in the offspring of one parent with schizophrenia and healthy controls are summarized in Table 2. Based on the obtained results shown in Table 2, the comparison of the $\mathrm{CBCL}$ score in primary school subjects demonstrated a significant difference between the two groups $(P=0.003)$. On the other hand, the $\mathrm{CBCL}$ score of teenagers was not significantly different between the two groups $(P=0.06)$. Moreover, the $C B C L$ score of primary school girls $(P=0.03)$ and boys $(P=$ 0.04 ) was found to be significantly different between the two study groups. However, no significant difference was reported in the $C B C L$ score of teenage girls $(P=0.09)$ and teenage boys $(P=0.09)$.

The mean of behavioral problems score was $55.89 \pm$ 11.89 in boys with a schizophrenic parent and $56.9 \pm$ 11.12 in girls with a schizophrenic parent. According to the Kolmogorov-Smirnov test, the distribution of behavioral problems score was normal $(Z=0.7 ; P>0.005)$. In addition, the homoscedasticity of the $C B C L$ score was confirmed ( $F=0.19 ; P>0.005)$. The evaluation of the $\mathrm{CBCL}$ score in two genders indicated that the two groups were not significantly different $(t=-0.78 ; P=0.21$ ).

The mean $C B C L$ score in all primary school and teenage subjects was $55.69 \pm 10.34$ and $57.42 \pm 12.11$, respectively. The behavioral problem score was indicated to have a normal distribution $(Z=0.59 ; P>0.005)$ and was homogeneous ( $F=0.89 ; P>0.005)$. The assessment of the $C B C L$ score in the two age ranges showed that there was no significant difference between the two study groups $(t=-0.91 ; P=0.13)$.

Table 1. Frequency of age and gender in the two study groups

\begin{tabular}{|c|c|c|c|c|c|}
\hline \multirow[t]{2}{*}{ Variables } & & \multicolumn{2}{|c|}{$\begin{array}{c}\text { Children with schizophrenic } \\
\text { parent }\end{array}$} & \multicolumn{2}{|c|}{ Children with healthy parent } \\
\hline & & Number & Percent & Number & Percent \\
\hline \multirow[t]{2}{*}{ Age range } & Middle childhood & 33 & 55 & 38 & 63.3 \\
\hline & Adolescence & 27 & 45 & 22 & 36.7 \\
\hline \multirow[t]{2}{*}{ Gender } & Male & 30 & 50 & 35 & 58.3 \\
\hline & Female & 30 & 50 & 25 & 41.7 \\
\hline
\end{tabular}


Table 2. Comparison of CBCL score based on gender in the offspring of one parent with schizophrenia and healthy controls

\begin{tabular}{|c|c|c|c|c|c|c|c|c|c|c|c|}
\hline \multirow{3}{*}{\multicolumn{2}{|c|}{ CBCL score }} & \multirow{2}{*}{\multicolumn{2}{|c|}{$\begin{array}{l}\text { Offspring of } \\
\text { one parent } \\
\text { with } \\
\text { schizophrenia }\end{array}$}} & \multirow{2}{*}{\multicolumn{2}{|c|}{$\begin{array}{l}\text { Healthy } \\
\text { parents }\end{array}$}} & \multicolumn{2}{|c|}{ Normality } & \multirow{3}{*}{$\mathbf{F}$} & \multirow{3}{*}{ P-value } & \multicolumn{2}{|c|}{$\begin{array}{l}\text { Comparison } \\
\text { two groups }\end{array}$} \\
\hline & & & & & & Z & P-value & & & $t$ & P-value \\
\hline & & Mean & SD & Mean & SD & & & & & & \\
\hline \multicolumn{2}{|c|}{ Middle childhood } & 60.73 & 10.34 & 50.66 & 9.49 & 0.57 & $<0.005$ & 0.61 & $<0.005$ & 0.46 & 0.003 \\
\hline \multicolumn{2}{|c|}{ Adolescence } & 62.28 & 11.73 & 52.56 & 12.54 & 0.89 & $<0.005$ & 0.08 & $<0.005$ & 1.91 & 0.06 \\
\hline \multirow[t]{2}{*}{$\frac{n}{i \frac{\pi}{i}}$} & $\begin{array}{l}\text { Middle } \\
\text { childhood }\end{array}$ & 61.02 & 10.78 & 50.09 & 11.79 & 0.98 & $<0.005$ & 0.34 & $<0.005$ & 3.34 & 0.03 \\
\hline & Adolescence & 64.23 & 10.56 & 53.11 & 12.01 & & & & & 1.98 & 0.09 \\
\hline \multirow[t]{2}{*}{ ๙ิ } & $\begin{array}{l}\text { Middle } \\
\text { childhood }\end{array}$ & 60.43 & 11.13 & 51.23 & 8.98 & 0.79 & $<0.005$ & 1.32 & $<0.005$ & 2.95 & 0.04 \\
\hline & Adolescence & 66.33 & 12.98 & 54.01 & 13.09 & 0.82 & $<0.005$ & 0.09 & $<0.005$ & 1.71 & 0.09 \\
\hline
\end{tabular}

\section{Discussion}

According to the results obtained in the present study, the behavioral problem of primary school children with one schizophrenic parent was higher than children with healthy parents. This was confirmed when the behavioral problem of primary school children was compared to the control group separately in boys and girls. However, no difference was observed concerning the behavioral problems among teenage participants. Our findings regarding the high frequency of aggression items in primary school children with schizophrenic parents, compared to the individuals with normal parents are consistent with other similar studies [16].

The effects of psychotic disorders of parents on the behavioral problems of children were assessed in some studies [17]. Donatelli et al. [18] reported a higher rate of behavioral problems in primary school offspring of parents with psychosis, in comparison with the offspring of unaffected parents, which is in line with our results. However, they showed a different outcome in younger children aged about 4 years. In addition, these authors observed no difference between the behavior problems of children with psychotic parents and children with normal parents. This may be due to the lack of variability in the results obtained from younger children.

These findings were confirmed in a prospective cohort study performed by Bearden et al., which evaluated the deviant behaviors of pre-schizophrenia subjects at ages 4 and 7 years. They found no relationship in terms of social maladjustment between pre-schizophrenia subjects at the age of 4 years. However, social maladjustment was a predictor for adult schizophrenia in seven-year-old children [19]. The observed difference can be attributed to the increasing expectation of community from older children regarding normal behavior. Therefore, behavioral problems elevate from early to later childhood in pre-schizophrenia individuals.

There is a diverse pattern for the gender and type of parental psychosis in children with behavioral problems. Donatelli et al. [18] showed a high risk for externalizing behaviors during the middle childhood period of children with parents affected by schizophrenia and other non-affective psychoses. Furthermore, the offspring of parents with affective psychoses are at high risk for internalizing behaviors.

The latter finding is in line with other similar studies conducted by Weintraub and Neale [16]. These investigations demonstrate a difference in the aggressiveness items of children with schizophrenic parents, compared to children with normal parents.

To our knowledge, the internalizing and externalizing problems are different in normative samples. The internalizing problems occur more in girls than boys with non-psychotic parents. This difference was not observed in high-risk children and the incidence of internalizing problems in this group was higher in boys than girls. Moreover, a gender difference was observed in 7-year-old children when comparing the high-risk parents versus normal-risk parents.

The rate of externalizing problems was higher in high-risk girls than normal-risk girls with normal parents. On the other hand, the rate of internalizing problems was higher among high-risk male children than normal-risk girls [20]. These findings on childhood behavioral problems can reveal the heritable phenotype of schizophrenia or reflect the early expressions of gender differences [21]. 
Goldstein et al. found gender-related differences among the relatives of people with schizophrenia, such as exhibiting, more irritability, and emotional instability in females and displaying more flat affect in males [22]. However, social anxiety disorder and other issues, including alterations of brain structure and function are not always observed in the relatives of people with schizophrenia [23].

Moreover, some traits, such as explosiveness, agitation, overt hostility, sexual delusion, and affective symptoms occurred more in females than males. However, some other traits, including withdrawal, passiveness, isolation, and less exhibitionism, hostility, and impulsiveness were more observed among men with schizophrenia than women [24]. Although in our study the behavior problems were different among girls and boys in middle childhood, no difference was reported among teenagers of both genders. These findings were inconsistent with other similar studies [25] that emphasize the effect of psychotic diseases of parents on the behavioral problems of adolescents. It is possible that the low sample size affected our outcome. Future studies with a larger sample size are suggested to obtain more accurate results.

Different results between middle childhood and adolescence among the offspring of one parent with schizophrenia and healthy controls may indicate the higher influence of other relationships with friends, classmates, and other individuals on the mental health of teenagers. The interaction between parents' and children's behaviors, in addition to the contributory role of familial factors to the development of schizophrenia-like disorders, have been reported in some studies. An association between the pathologic affective style and a benign outcome was observed in a study carried out by Doan et al. [26]. The deviation of parental communication and affective styles of communication plays an important role in the formation of psychopathic and behavioral disorders in children and adolescents. In a study completed by Forsyth et al. [27], 1070 offspring at low risk for schizophrenia and 373 offspring at high risk for schizophrenia were assessed. The obtained results showed that the high-risk children had weaker educational performance, compared to the low-risk subjects.

According to the literature, the offspring of one parent with severe mental diseases, such as schizophrenia have low self-esteem [13]. These children have low emotional security and suffer the lack of intimacy in the family environment leading to inconsistency, deviation, and inappropriate performance. Moreover, parenting and socialization practices among parents with schizophrenia are other reasons for higher rates of externalizing and internalizing problems in high-risk children [28].
Moreover, Erlenmeyer-Kimling et al. [29] demonstrated that the offspring of schizophrenic parents have dysfunctions in several neurobehavioral domains during childhood or adolescence. These dysfunctions have been reported in the offspring of parents with other psychiatric disorders. In addition, deficits in the offspring of schizophrenic parents in terms of neuromotor and cognitive (especially attentional and memory) functions were revealed in some studies [30].

In conclusion, the results of this study add to the evidence that indicated the children and adolescents of parents with various forms of psychosis are at risk for behavioral problems. Longitudinal studies examining the relationship of behavior problems during early childhood with psychologic disorders in adolescence and adulthood can provide valuable information about the course and predictive validity of early childhood difficulties. Therefore, these studies are beneficial in the early identification of these problems and are useful in the development of intervention programs.

According to the finding of the present study, it can be concluded that school children with a parent suffering from schizophrenia have more behavioral problems than those with normal parents. These findings represent the role of the mental health of parents in prevention from developing behavioral problems in children.

Consequently, training concerning raising children for parents whose partner is suffering from serious mental disorder seems to be essential. Furthermore, some programs should be taken into consideration in the future for training children and teenagers with schizophrenic parents to improve health mentality in these individuals.

\section{Advantages and limitations}

The main strength of this study was the assessment of behavioral problems in the two age groups of adolescence and childhood by psychologists who were blind to parental psychiatric status. However, our sample size was not enough for generalizing and confirming our hypotheses and our samples were not selected randomly. In addition, the outcome may be affected by the uncontrolled intervention variables. It is possible that the researcher may have not been able to provide a complete picture of the mental illness of parents and the effects on the mental health of children. The investigation of other factors influencing the behavioral problems in children and adolescents, such as the physical condition, socioeconomic status, official and informal social support, family status, and diverse personality variables are recommended for future longitudinal studies with larger sample size. Our findings demonstrated that primary school children with a parent suffering from schizophrenia have more 
behavioral problems than those with normal parents. However, this result was not observed in the group of adolescents.

\section{Conclusion}

In summary, the findings of this study can be regarded as evidence supporting the impact of psychotic problems, including schizophrenia in parents on the behavioral problems of children.

\section{Acknowledgments}

The current study is based on a thesis approved by the Islamic Azad University of Isfahan (Khorasgan). The authors would like to extend their gratitude toward the participants and all the individuals who assisted in this research.

\section{References:}

1. Casas F, Coenders G, González M, et al. Testing the relationship between parents' and their children's subjective well-being. Journa of Happiness Studies. 2011; 13(6): 1031-1051, doi: 10.1007/ s10902-011-9305-3.

2. van Eijck FE, Branje SJT, Hale WW, et al. Longitudinal associations between perceived parent-child relationship quality and depressive symptoms in adolescence. J Abnorm Child Psychol. 2010; 38(6): 751-763, doi: 10.1007/s10802-010-9401-6, indexed in Pubmed: 20217211.

3. Eichelsheim VI, Buist KL, Deković $\mathrm{M}$, et al. Associations among the parent-adolescent relationship, aggression and delinquency in different ethnic groups: a replication across two Dutch samples. Soc Psychiatry Psychiatr Epidemiol. 2010; 45(3): 293-300, doi: 10.1007/ s00127-009-0071-z, indexed in Pubmed: 19466371.

4. Reynolds C. Behavior assessment system for children. The Corsini Encyclopedia of Psychology. 2010, doi: 10.1002/9780470479216. corpsy0114.

5. Astill RG, Van der Heijden $\mathrm{KB}$, Van lizendoorn $\mathbf{M H}$, et al. Sleep, cognition, and behavioral problems in school-age children: a century of research meta-analyzed. Psychol Bull. 2012; 138(6): 1109-1138, doi: 10.1037/a0028204, indexed in Pubmed: 22545685

6. Neece CL, Green SA, Baker BL. Parenting stress and child behavior problems: a transactional relationship across time. Am J Intellect Dev Disabil. 2012; 117(1): 48-66, doi: 10.1352/1944-7558-117.1.48, indexed in Pubmed: 22264112.

7. Gross HE, Shaw DS, Moilanen KL. Reciprocal associations between boys' externalizing problems and mothers' depressive symptoms. J Abnorm Child Psychol. 2008; 36(5): 693-709, doi: 10.1007/ s10802-008-9224-x, indexed in Pubmed: 18288602

8. Van de, Spruijt A, De Go, et al. longitudinal study on transactional relations between parental marital distress and adolescent emotional adjustment. Adolescence. 2007; 42: 115-36.

9. Brody $\mathrm{GH}, \mathrm{Ge} X$. Linking parenting processes and self-regulation to psychological functioning and alcohol use during early adolescence. J Fam Psychol. 2001; 15(1): 82-94, doi: 10.1037//08933200.15.1.82, indexed in Pubmed: 11322087.

10. Holmes J. John bowlby and attachment theory. Taylor \& Francis 2006

11. Krumwiede A. Attachment Theory According to John Bowlby and Mary Ainsworth: GRIN Verlag; 2014.

12. Association AP. Diagnostic and Statistical Manual of Mental Disorders (DSM-5®): American Psychiatric Publishing; 2013.

13. Nishida A, Sasaki T, Harada S, et al. Risk of developing schizophrenia among Japanese high-risk offspring of affected parent: outcome of a twenty-four-year follow up. Psychiatry Clin Neurosci. 2009;
63(1): 88-92, doi: 10.1111/j.1440-1819.2008.01892.x, indexed in Pubmed: 19067993.

14. Helton J, Vaughn M, Kavish N, Boutwell BB. The Association of Externalizing and Internalizing Problems with Indicators of Intelligence in a Sample of At-Risk Children. bioRxiv. 2018: 210-500.

15. Moharreri F, Habrani P, Heidari Yazdi A. Epidemiological survey of psychiatric disorders in children and adolescents of Mashhad in 2009. Journal of Fundamentals of Mental Health. 2015; 17(5): 247-253.

16. Weintraub S. Risk factors in schizophrenia: The Stony Brook high-risk project. Schizophrenia Bulletin. 1987; 13(3): 439-450.

17. Boričević Maršanić $V$, Aukst Margetić $B$, Jukić $V$, et al. Self-reported emotional and behavioral symptoms, parent-adolescent bonding and family functioning in clinically referred adolescent offspring of Croatian PTSD war veterans. Eur Child Adolesc Psychiatry. 2014; 23(5): 295-306, doi: 10.1007/s00787-013-0462-2, indexed in Pubmed: 23949102

18. Donatelli JAL, Seidman $\sqcup$, Goldstein JM, et al. Children of parents with affective and nonaffective psychoses: a longitudinal study of behavior problems. Am J Psychiatry. 2010; 167(11): 1331-1338, doi: 10.1176/appi.ajp.2010.09020241, indexed in Pubmed: 20843870.

19. Bearden CE, Rosso IM, Hollister JM, et al. A prospective cohort study of childhood behavioral deviance and language abnormalities as predictors of adult schizophrenia. Schizophr Bull. 2000; 26(2): 395-410, doi: 10.1093/oxfordjournals.schbul.a033461, indexed in Pubmed: 10885639

20. Zahn-Waxler C, Crick N, Shirtcliff E, et al. The Origins and Development of Psychopathology in Females and Males. Developmental Psychopathology. 2015: 76-138, doi: 10.1002/9780470939383.ch4.

21. Goldstein JM, Walder DJ. Sex differences in schizophrenia: the case for developmental origins and etiological implications. The early course of schizophrenia. 2006: 147-73

22. Goldstein JM, Faraone SV, Chen WJ, et al. Sex differences in the familial transmission of schizophrenia. Br J Psychiatry. 1990; 156(2): 819-826, doi: 10.1192/bjp.156.6.819, indexed in Pubmed: 2207512

23. Seidman $\sqcup$, Thermenos HW, Koch JK, et al. Altered brain activation in dorsolateral prefrontal cortex in adolescents and young adults at genetic risk for schizophrenia: an fMRI study of working memory. Schizophrenia Research. 2006; 85((1-3)): 58-72.

24. Goldstein JM, Link BG. Gender and the expression of schizophrenia. J Psychiatr Res. 1988; 22(2): 141-155, doi: 10.1016/00223956(88)90078-7, indexed in Pubmed: 3404482

25. Sørensen HJ, Mortensen EL, Reinisch JM, et al. Parental psychiatric hospitalisation and offspring schizophrenia. World J Biol Psychiatry. 2009; 10(4 Pt 2): 571-575, doi: 10.1080/15622970701472078, indexed in Pubmed: 17853283.

26. Doane JA, West KL, Goldstein MJ, et al. Parental communication deviance and affective style. Predictors of subsequent schizophrenia spectrum disorders in vulnerable adolescents. Arch Gen Psychiatry. 1981: 38(6): 679-685, doi: 10.1001/archpsyc. 1981.01780310079008, indexed in Pubmed: 7247631.

27. Forsyth JK, Ellman LM, Tanskanen A, et al. Genetic risk for schizophrenia, obstetric complications, and adolescent school outcome: evidence for gene-environment interaction. Schizophr Bull. 2013; 39(5): 1067-1076, doi: 10.1093/schbul/sbs098, indexed in Pubmed: 22941745

28. Stromwall LK, Robinson EA. When a family member has a schizophrenic disorder: practice issues across the family life cycle. Am J Orthopsychiatry. 1998; 68(4): 580-589, doi: 10.1037/h0080366, indexed in Pubmed: 9809117.

29. Erlenmeyer-Kimling L. Neurobehavioral deficits in offspring of schizophrenic parents: liability indicators and predictors of illness. Am J Med Genet. 2000; 97(1): 65-71, doi: 10.1002/(sici)10968628(200021)97:1<65::aid-ajmg9>3.0.co;2-v, indexed in Pubmed: 10813806

30. Jokiranta E, Brown AS, Heinimaa M, et al. Parental psychiatric disorders and autism spectrum disorders. Psychiatry Res. 2013. 207(3): 203-211, doi: 10.1016/j.psychres.2013.01.005, indexed in Pubmed: 23391634 